\title{
TV/Series
}

18 | 2020

Séries et espace

\section{Espaces fictionnels, espaces interactionnels : approches linguistiques des séries}

\section{Camille Debras}

\section{(2) OpenEdition}

\author{
Journals
}

Édition électronique

URL : http://journals.openedition.org/tvseries/4462

DOI : 10.4000/tvseries.4462

ISSN : 2266-0909

\section{Éditeur}

GRIC - Groupe de recherche Identités et Cultures

\section{Référence électronique}

Camille Debras, «Espaces fictionnels, espaces interactionnels : approches linguistiques des séries », TV/Series [En ligne], 18 | 2020, mis en ligne le 15 septembre 2020, consulté le 08 décembre 2020. URL : http://journals.openedition.org/tvseries/4462 ; DOI : https://doi.org/10.4000/tvseries.4462

Ce document a été généré automatiquement le 8 décembre 2020

\section{(c) (i) (3) $\Theta$}

TV/Series est mis à disposition selon les termes de la licence Creative Commons Attribution - Pas d'Utilisation Commerciale - Pas de Modification 4.0 International 


\title{
Espaces fictionnels, espaces interactionnels : approches linguistiques des séries
}

\author{
Camille Debras
}

1 Les chercheurs en linguistique de l'oral font face à un défi constant : celui de collecter des corpus de données orales pour leurs analyses. Transcrire des données d'interaction orale ou obtenir les droits de les étudier et éventuellement de les partager avec la communauté des chercheurs est une tâche longue et complexe. Pour leurs premiers travaux de jeunes chercheurs, les masterants se retrouvent parfois devant une véritable gageure lorsqu'il s'agit, en une seule année universitaire, de collecter un corpus original de données orales, le transcrire, et en proposer une analyse en linguistique interactionnelle et/ou multimodale (pragmatique; syntaxe de l'oral; grammaire multimodale par exemple). En accord avec leurs directeurs de recherche, ils peuvent choisir de faire porter leur étude de la langue orale sur des séries télévisées. Celles-ci ont en effet l'intérêt majeur de constituer, jusqu'à un certain point, des corpus clé en main : les enregistrements sont déjà réalisés et faciles d'accès ; une quantité non négligeable de données langagières peut être rassemblée lorsqu'un grand nombre d'épisodes existe; des transcriptions de qualité sont, souvent, déjà disponibles sur internet, où nombre de fans les réalisent bénévolement et les mettent en ligne sur des sites dédiés à leurs séries préférées. Souvent, cependant, il arrive que ces travaux de jeunes chercheurs ne problématisent pas assez la nature même des corpus interactionnels fictionnels, et proposent hâtivement des conclusions sur le fonctionnement de la langue interaction, sans prendre en compte les spécificités d'un corpus de fiction.

2 Cet article est conçu comme une base de référence pour les travaux de linguistique de l'oral s'appuyant sur des corpus fictionnels comme les séries. Dans cet article, nous proposons de montrer, à l'appui d'un tour d'horizon d'études linguistiques sur les séries, que les pratiques langagières dans les séries ne correspondent pas exactement aux pratiques langagières du monde réel. Nous souhaitons montrer que l'analyse 
linguistique des séries est un champ d'étude riche et passionnant, mais que pour autant, les pratiques langagières des séries doivent être étudiées pour ce qu'elles sont des interactions fictionnelles dont le destinataire réel est le spectateur - car leur nature leur confère des spécificités propres, qui les distinguent à certains égards des pratiques langagières observables dans le monde réel.

\section{L'interaction médiatisée : un espace à deux niveaux}

3 La notion d'espace est centrale pour toute approche linguistique qui théorise le contexte d'un énoncé. En pragmatique, en sociolinguistique interactionnelle, en analyse conversationnelle ou en anthropologie linguistique, pour citer les principales approches intéressées à la linguistique de l'oral, les pratiques langagières situées, c'està-dire indissociables d'une situation sociale et spatiale ${ }^{1}$. L'espace interactionnel peut être défini comme l'espace physique d'interaction et d'interlocution où les participants ont des rôles et des statuts qu'ils co-construisent par le biais de leurs pratiques langagières.

4 Par essence, la parole médiatisée (des entretiens télévisés aux fictions d'écran) s'inscrit dans l'enchâssement d'au moins deux espaces interactionnels : d'une part, l'interaction entre les participants à l'écran, et de l'autre, l'interaction entre le contenu porté à l'écran et le spectateur qui le visionne ${ }^{2}$. Dès 1984, Burger ${ }^{3}$ distingue deux cercles de communication: un premier cercle («primary situation»/ «inner circle»), où le dialogue a lieu, et un second cercle (« secondary situation»/ « outer circle»), celui de la relation entre les participants du dialogue et le public devant l'écran. Les théorisations plus récentes de cette double articulation ${ }^{4}$, ou double-layering ${ }^{5}$ renversent la hiérarchie entre ces deux niveaux, car l'adresse au (télé)spectateur est bien la visée ultime de la parole médiatisée. Dans le cas particulier des séries, ces deux niveaux ou espaces sont de nature différente: l'espace interactionnel fictionnel entre les participants est plus ou moins le miroir d'interactions réelles, tandis que l'espace interactionnel entre le spectateur et la fiction est bien réel ${ }^{6}$.

\section{Approches linguistiques des séries : quoi, pourquoi et comment?}

5 L'étude linguistique des séries est riche d'enjeux pour les études culturelles et en particulier les études anglophones, et ce pour un grand nombre de raisons. Tout d'abord, puisqu'une grande partie des séries consommées par les spectateurs dans le monde entier sont produites aux États-Unis, on peut légitimement s'attendre à ce que celles-ci exercent une influence sur les pratiques langagières et l'attitude de millions de personnes dans le monde ${ }^{7}$. À cette idée, Bednarek ${ }^{8}$ ajoute trois motivations supplémentaires pour le linguiste en études anglophones: les séries sont des productions culturelles au même titre que les romans et les films; leurs contenus langagiers sont susceptibles d'avoir un impact non négligeable sur les apprenants de la langue utilisée, par exemple l'anglais; enfin, elles peuvent servir de support pour l'apprentissage dans la classe de langue ${ }^{9}$.

6 Les séries sont, par essence, un objet complexe et multimodal qui utilise plusieurs modalités sémiotiques, dont le discours, pour la construction du sens. Aucune approche ne peut saisir totalement cette complexité ${ }^{10}$, et nombre de chercheurs dans le domaine des études télévisuelles sont parfaitement conscients des limites de leur approche et 
tentent d'en prendre en compte les faiblesses, qu'il s'agisse d'approches plutôt "logocentrées" en stylistique ${ }^{11}$ ou plutôt "vidéocentrées » en études filmiques ${ }^{12}$. Si toute approche se révèle forcément incomplète, la possibilité de choisir une approche centrée sur le langage est tout à fait légitime, tant qu'elle reste consciente de ses propres limites, ainsi que de la complémentarité des modalités de construction du sens ${ }^{13}$.

7 Piazza, Bednarek et Rossi ${ }^{14}$ identifient quatre grands enjeux pour l'analyse linguistique des films et des séries :

1. l'interface entre les discours visuel et verbal,

2. le rapport entre vie réelle et discours fictionnel, autrement dit, la question de la représentation,

3. la définition des personnages,

4. et la relation entre les participants représentés et réels, c'est-à-dire entre la fiction et le spectateur.

8 Cet article propose de laisser de côté le premier point sur la question de la construction multimodale du sens, qui mérite à elle seule d'amples développements, pour se concentrer sur les points 2 à 4 qui seront traités successivement.

\section{Interaction fictionnelle, miroir de l'interaction réelle?}

9 La question du réalisme des pratiques langagières interactionnelles des personnages de séries se pose autant pour les professionnels de l'écriture scénaristiques que pour les linguistes. Dans son manuel de 1984 The Screenwriter's Workbook ${ }^{15}$, Syd Field conseille aux apprentis scénariste de s'inspirer d'enregistrements de conversations réelles pour écrire des dialogues :

Tape a conversation with a friend or acquaintance. Play it back and listen to it. Notice how fragmented it is, how quickly thoughts come and go. If you want to see what "real" dialogue looks like, type it up in screenplay form. Listen for mannerisms, and inflections, find the style of speech, the phrasing. Then think about your character speaking in those "rhythms," or in that "language" 16.

10 La question de savoir si les personnages des séries parlent comme leurs spectateurs est au cœur du livre de Paul Quaglio, Televison Dialogue: The Sitcom Friends vs. Natural Conversation $^{17}$, qui utilise les outils de la linguistique de corpus pour comparer le genre discursif du dialogue de la fiction télévisée Friends (NBC, 1994-2004) à celui de la conversation spontanée.

Le constat initial de Quaglio est celui d'un consensus qu'il observe, sur l'idée que le personnage doit être un miroir tendu au spectateur, notamment dans sa manière de parler, pour permettre au spectateur de s'identifier :

There seems to be an agreement among scholars that, despite the natural restrictions imposed by the televised medium, television dialogue should sound natural; otherwise, viewer identification with the show characters can be negatively impacted, thus, potentially, affecting the success of the show ${ }^{18}$.

Douglas Biber ${ }^{19}$ ouvre son avant-propos du livre de Quaglio sur l'idée que les pratiques langagières des séries semblent être le reflet exact de celles du monde réel :

Don't we talk just like people on television? Or rather, don't those people talk just like us? Conversations on television seem completely natural to the normal viewer. But is that because we have come to expect a particular style of interaction on TV, 
or because those interactions accurately capture the actual linguistic characteristics of everyday conversation?

Corpus-based analysis is ideally suited for a research question of this type [... $]^{2021}$.

13 Par leurs études, Quaglio ${ }^{22}$ et Bednare ${ }^{23}$ montrent que par bien des aspects, le genre discursif de l'oral scripté des séries est proche de la conversation spontanée. Pour ce faire, ils comparent de grands corpus de transcriptions de conversations ordinaires avec des transcriptions de dialogues de séries. Il ne s'agit pas des scripts originaux, mais de fan transcripts, qui sont des transcriptions réalisées et mises gratuitement à disposition par des fans sur le net. Ces transcriptions amateures sont, selon ces linguistes, particulièrement fiables et détaillées ${ }^{24}$. Quaglio compare le corpus de 600,000 mots de neuf des dix saisons de Friends (1994-2003) à l'American Conversation Subcorpus $\mathrm{du}$ Longman Grammar Corpus (ASCLGC) (4,000,000 mots environ). Ce corpus d'enregistrements audio a été construit de manière à représenter le genre de la conversation en anglais aux États-Unis, et inclut une variété de contextes (espaces publics, salles de classes, maisons), de types d'interactions (conversations informelles, en face à face ou au téléphone), de régions géographiques, et de profils de locuteurs (âge, sexe, emploi, formation) ${ }^{25}$. Par comparaison, le corpus de transcriptions de la série Friends est bien plus court, et présente un nombre plus restreint de contextes, de types et de sujets d'interactions, se limitant principalement aux thèmes de l'amitié, la rencontre amoureuse et la sexualité.

Sur le plan méthodologique, Quaglio procède tout d'abord à la normalisation des fréquences pour obtenir le ratio du type pour 1 million de mots, puis à un étiquetage morpho-syntaxique automatique (POS (part-of-speech) tagging) et détermine les marqueurs qu'il souhaite observer. Il s'appuie ensuite sur des tests statistiques de comparaison entre les deux corpus, pour déterminer si les fréquences comparées sont statistiquement signifiantes (c'est-à-dire, si les résultats sont généralisables ou non). Il sélectionne des formes considérées comme typiques d'une caractéristique, (voir la liste de formes prises en compte pour l'étude du caractère abstrait du discours, représentée dans la partie gauche de la Figure 1). Puis, grâce à un concordancier, il procède à l'analyse détaillée systématique des sens exprimés par une forme (représentée dans la partie droite de la Figure 1).

Figure 1 : liste de marqueurs d'un trait discursif comme le vague (à gauche); analyse par concordancier (pour le marqueur kind of, à droite).

\begin{tabular}{|c|c|c|c|c|}
\hline Categories & Feature & Conversation & Friends & Similar \\
\hline Hedges & $\begin{array}{l}\text { Kind of (like) } \\
\text { Sort of (like) }\end{array}$ & : & & \\
\hline Vague coordination tags & $\begin{array}{l}\text { Or something (like that) } \\
\text { Or anything (like that) } \\
\text { (and) stuff (like that) }\end{array}$ & $\vdots$ & & \\
\hline Nouns of vague reference & $\begin{array}{l}\text { Thing(s) } \\
\text { Stuff } \\
\text { Shit }\end{array}$ & $\vdots$ & & \\
\hline Discourse markers & $\begin{array}{l}\text { You know } \\
\text { I mean }\end{array}$ & . & & - \\
\hline Stance markers & $\begin{array}{l}\text { Probably } \\
\text { Perhaps } \\
\text { Maybe }\end{array}$ & . & : & \\
\hline Modals & $\begin{array}{l}\text { Could } \\
\text { Might }\end{array}$ & - & & - \\
\hline Copular verbs & $\begin{array}{l}\text { Seem } \\
\text { Appear }\end{array}$ & & & : \\
\hline Utterance final so & So & . & & \\
\hline
\end{tabular}

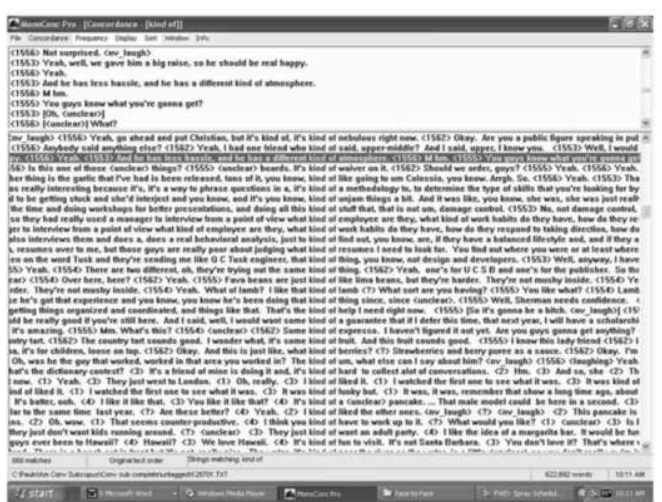


Les conclusions de Quaglio sont les suivantes : le contenu discursif de Friends présente les mêmes traits principaux que la conversation spontanée. Les marques les plus fréquentes sont les pronoms de première et de deuxième personnes, les verbes d'états mentaux (want, know, think, feel), les contractions, le temps présent, les particules de discours (Oh, Okay) et les prépositions séparées de leur objet (preposition stranding, par exemple: the person I'm thinking of). Cependant, il relève également des différences fondamentales : les conversations dans Friends sont moins imprécises, moins narratives, plus émotionnelles et plus informelles que la conversation spontanée. Voici le résumé de son étude :

1. Friends shares the core linguistic features that characterize natural conversation;

2. Vague language is much more pervasive in natural conversation than in Friends;

3. Friends presents higher frequencies of linguistic features marking emotional language;

4. Friends presents higher frequencies of linguistic features marking informality;

5. Natural conversation has a higher degree of narrativeness when compared to Friends;

6. Some differences between the two corpora are due to restrictions and/or influences of the televised medium ${ }^{26}$.

16 L'étude de Bednarek ${ }^{27}$ propose une recherche semblable, cette fois à partir de la série Gilmore Girls (The WB/The CW, 2000-2007). Bednarek compare les fan transcripts de la série Gilmore Girls avec plusieurs corpus, notamment un corpus de transcriptions de conversations spontanées, un corpus écrit, et un corpus de fans dans le but de déterminer si (i) les caractéristiques formelles de l'oral scripté le rapprochent plus de l'oral ou de l'écrit et (ii) si la série est bien représentative du genre de l'oral scripté des séries. Le corpus de fan transcripts de Gilmore Girls (GiGi) qu'elle a rassemblé et manuellement corrigé ( 7 saisons de 2000 à $2007 ; 153$ épisodes ; 1,1 million de mots) est comparé notamment au Longman Spoken American Corpus (LSAC, 4,8 millions de mots), la composante écrite de l'American National Corpus (18 millions de mots), et à un corpus de fan transcripts d'autres séries (TV Corpus, incluant des transcriptions d'épisodes de NCIS, Bones, House, Lost notamment: 220000 mots). Au plan méthodologique, elle s'appuie sur la comparaison des suites de mots les plus fréquentes dans chaque corpus, appelés $n$-grams ou lexical bundles), car ceux-ci permettent de distinguer les genres de texte (en particulier les suites de quatre mots) ${ }^{28}$. Son analyse montre que le corpus GiGi est plus proche du TV Corpus que du corpus d'oral LSAC, mais reste cependant bien plus proche du LSAC que du corpus écrit. Plus précisément, il existe donc un genre discursif spécifique du dialogue fictionnel des séries ; que celui-ci se rapproche du genre discursif de l'oral spontané, mais qu'il s'en distingue car il est plus plus émotionnel, moins narratif et moins vague; ses résultats confirment donc ceux de Quaglio. Elle en conclut notamment que les scénaristes peinent à imiter la conversation spontanée :

In other words, GiGi and the TV corpus are indeed more similar to each other than GiGi and LSAC, showing that while the scripted language of GiGi does have similarities with unscripted spoken American English, there are limitations in how far scriptwriters and actors are able to mirror 'naturally occurring' language ${ }^{29}$.

17 Cette idée est, bien sûr, comme le remarque Bednarek elle-même, à nuancer : le fait que le genre discursif des séries est moins imprécis, moins narratif et plus émotionnel a très probablement un lien direct avec la double-articulation spécifique à ce genre de corpus. En effet, l'implicite est vraisemblablement plus important entre les participants d'une conversation spontanée qui partagent une situation d'interaction et se connaissent, 
tandis que le contenu informationnel d'un échange fictionnel se doit d'être plus explicite, pour que le spectateur dispose de toutes les informations nécessaires pour en comprendre la teneur. Si la dimension narrative est moins forte dans les dialogues des séries, c'est très probablement parce qu'elle est transposée à la fiction même ${ }^{30}$. Enfin, la plus grande charge émotionnelle des dialogues télévisés est imputable à la dramatisation des événements représentés par la fiction.

Pour conclure sur ce point, ces deux études permettent à elles seules de montrer que les corpus de fiction télévisée constituent un genre discursif spécifique, qui ne coïncide pas exactement avec celui de la parole spontanée, même s'il s'en rapproche.

\section{Approche linguistique de la définition des personnages}

La question de la définition des personnages (terme que nous choisissons pour traduire la notion de characterization) est un deuxième enjeu central des approches linguistiques des séries. Nous axerons cette section sur l'étude proposée par Bednarek en 2011 intitulée "The stability of the televisual character: a corpus stylistic case study " Dans ce chapitre, Bednarek propose une étude stylistique de la définition des personnages ( «a corpus stylistic study of characterization », centrée sur le personnage principal de la comédie dramatique («dramedy») Gilmore Girls, Lorelai Gilmore, toujours à l'appui des outils de la linguistique de corpus. Bednarek part du constat qu'une différence fondamentale de la série, par rapport au film, est le temps long et la récurrence des épisodes. Chercheurs en études télévisuelles, professeurs d'écriture scénaristique et scénaristes s'accordent sur l'importance de la stabilité du personnage dans les séries télévisées, élément central pour la fidélisation du spectateur. Pour la spécialiste d'études télévisuelles R. Pearson, en effet,

The repetitive nature of the television series dictates a relative state of stability for its characters [...] In television, it's more accurate to talk about character accumulation and depth than it is to talk about character development ${ }^{32}$.

Selon le professeur d'écriture scénaristique Vorhaus, le public des séries, et en particulier des sitcoms, est en demande de retour du même :

Audiences return to a sitcom each week precisely to see their favourite characters

doing pretty much the same things they did last week and the week before that ${ }^{33}$.

21 Amy Sherman-Palladino, la créatrice et réalisatrice de Gilmore Girls expliquait également, en 2005, que le fait que le spectateur revit la même expérience chaque semaine est au cœur de sa série :

It's very important that it feel like the same show every week, because it's so verbal.

[...] It's really about people talking to each other and the way they talk to each

other, which is very specific. ${ }^{34}$

Bednare $\mathrm{k}^{35}$ propose une description linguistique de la stabilité du personnage de Lorelai Gilmore, à l'appui d'une méthode de linguistique de corpus appelées keyword / key cluster analysis. Keyword et key cluster correspondent respectivement à un mot ou groupe de mots dont la fréquence est inattendue dans un corpus, à l'appui d'un test statistique qui opère une comparaison avec un corpus de référence. La notion de keyness, c'est-à-dire la fréquence remarquable d'un mot, qui est ainsi identifié comme mot clé d'un corpus, est donc définie en termes de fréquence statistique inattendue. Le corpus testé par Bednarek rassemble l'ensemble des transcriptions des répliques du personnage de Lorelai Gilmore (fondées une fois encore sur des fan transcripts). La 
linguiste évalue la stabilité des pratiques langagières du personnage au plan diachronique (en recherchant d'éventuelles variations entre les saisons 1 à 7) ainsi qu'au plan intersubjectif (les keywords typiques du discours de Lorelai évoluent-ils selon le personnage à qui elle s'adresse ?).

Les résultats de son étude indiquent une grande stabilité linguistique du personnage à travers les 7 saisons : en effet, au plan diachronique, les mots-clés dont l'utilisation plus fréquente est statistiquement signifiante sont des noms propres d'autres personnages, ce qui indique une grande stabilité dans le style discursif du personnage par ailleurs. Cette stabilité diachronique est possiblement en lien avec le nombre limité de lieux et de situations typique de cette dramedy. A l'inverse, on peut remarquer que la stabilité de l'espace fictionnel, visible dans le maintien de lieux d'interaction, est également construite par la stabilité des pratiques langagières des personnages. Au plan intersubjectif, les résultats indiquent une variation limitée mais réelle, selon le personnage à qui Lorelai s'adresse. Les mots-clés identifiés indiquent par exemple le manque d'accommodation comme ressource humoristique, ou indexent le type de relations affectives entre les personnages. Bednarek montre en particulier comment les termes d'affection servent à la fois à construire le style du personnage, mais aussi les relations entre personnages, qui sont au centre de la série.

Cette méthode a bien évidemment ses limites, car elle ne permet pas, par exemple, de prendre en compte la complexité des pratiques langagières dans leur réalisation prosodique et/ou dans leur contexte multimodal et sémiotique. Elle permet, cependant de donner une vue d'ensemble globale des pratiques discursives d'un personnage, et de montrer, en l'occurrence, que la stabilité recherchée par la créatrice de la série est effectivement à l'œuvre. Comme le remarque Bednarek elle-même, la stabilité du personnage est en décalage notoire avec les conceptions postmodernes d'une identité changeante, fragmentée, dynamique, en construction. Cela peut s'expliquer par le fait que Gilmore Girls est une série mainstream, grand public, et peut-être fondée sur une conception occidentale plus traditionnelle de l'identité stable :

Further, the results may have to do with the fact that Gilmore Girls is a rather mainstream, conventional television series. The idea of a stable identity, which is at odds with postmodern conceptions of identity that emphasize the dynamic, localized, and fragmented aspects of identity construal (...), seem to sit well within "mainstream", popular and Western notions of identity. Such conceptions of the subject assume that "[h]uman beings are held to be fully centred and unified individuals with an inner core" (...). Traditional mainstream, as opposed to 'postmodern' texts, then, would not "threaten notions of the fully formed or whole "character" and would not construe "fluid, split or fractured 'subjects"' (...) but rather maintain character stability ${ }^{36}$.

\section{Screen-to-face interaction : I'interaction spectateur-fiction}

La stabilité du personnage de série, mise en évidence par les analyses linguistiques quantitatives, serait donc l'une des clés de la fidélité du spectateur, qui tisse un lien fort avec les personnages et l'univers fictionnel, au point d'y revenir au fil des épisodes. Mais quelle description linguistique peut-on donner de l'espace interactionnel où se joue la relation entre le spectateur et la série ? Des outils théoriques, notamment issus de la linguistique interactionnelle, permettent d'apporter des réponses à cette question. 
Short ${ }^{37}$ propose l'un des premiers modèles de l'interaction entre la fiction sur écran et le spectateur, à l'appui des théorisations classiques de la communication développées par Shannon et Weaver ${ }^{38}$. Pour ces derniers, la communication équivaut à la transmission d'un message par un émetteur à un récepteur par le biais d'un canal. Short adapte ce modèle théorique à la communication médiatisée par un écran, en proposant un modèle de discours enchâssé (embedded discourse), fondé sur une mise en abyme du dispositif de communication :

When we listen to two characters talking on stage we are meant to deduce, through what they say, what the author is telling us about them ${ }^{39}$.

Pour Short, ce dispositif est à l'origine de multiples effets, de l'ironie dramatique à la construction des personnages. La relation entre spectateur et fiction à l'écran se résumerait donc à l'adresse de l'auteur au spectateur par l'entremise des échanges entre les personnages, comme indiqué dans la figure 2.

Figure 2 : le modèle de mise en abyme du discours de Short ${ }^{40}$.

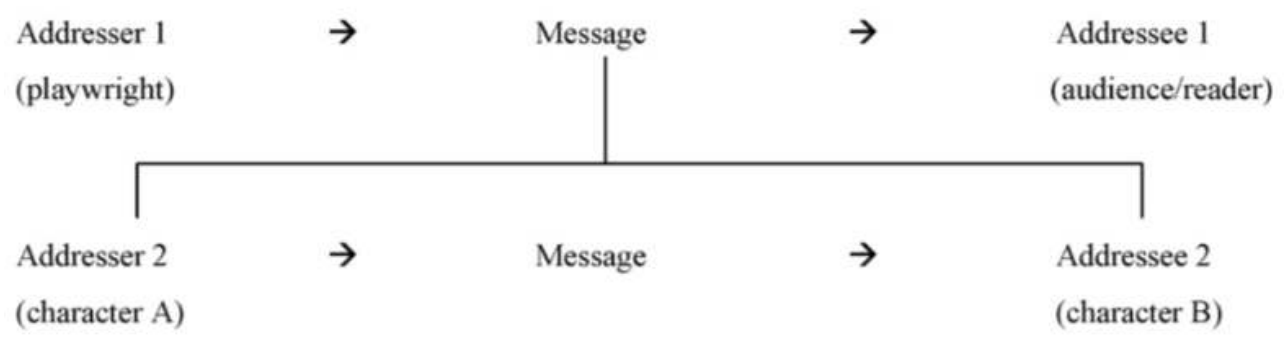

Cependant, les limites de ce type de théorisation se font rapidement jour. Tout d'abord, dans le cadre de la fiction à l'écran, l'auteur n'est pas identifiable à une personne unique: doit-on considérer que c'est le scénariste? le réalisateur? De plus, De nombreuses personnes prennent part à la construction du sens de la fiction : les acteurs eux-mêmes, ainsi que les auteurs, qui ne sont pas toujours les mêmes d'un épisode à l'autre. En outre, cette approche est trop simpliste, et donc insatisfaisante, car elle éclipse toute distinction, pourtant cruciale, entre auteur d'une part et narrateur ou point de vue adopté par l'œuvre de l'autre.

Le modèle proposé par Dynel ${ }^{41}$, reproduit dans la figure 3, pallie en partie les défauts du modèle de Short. 
Figure 3 : le modèle unidirectionnel de Dynel ${ }^{42}$.

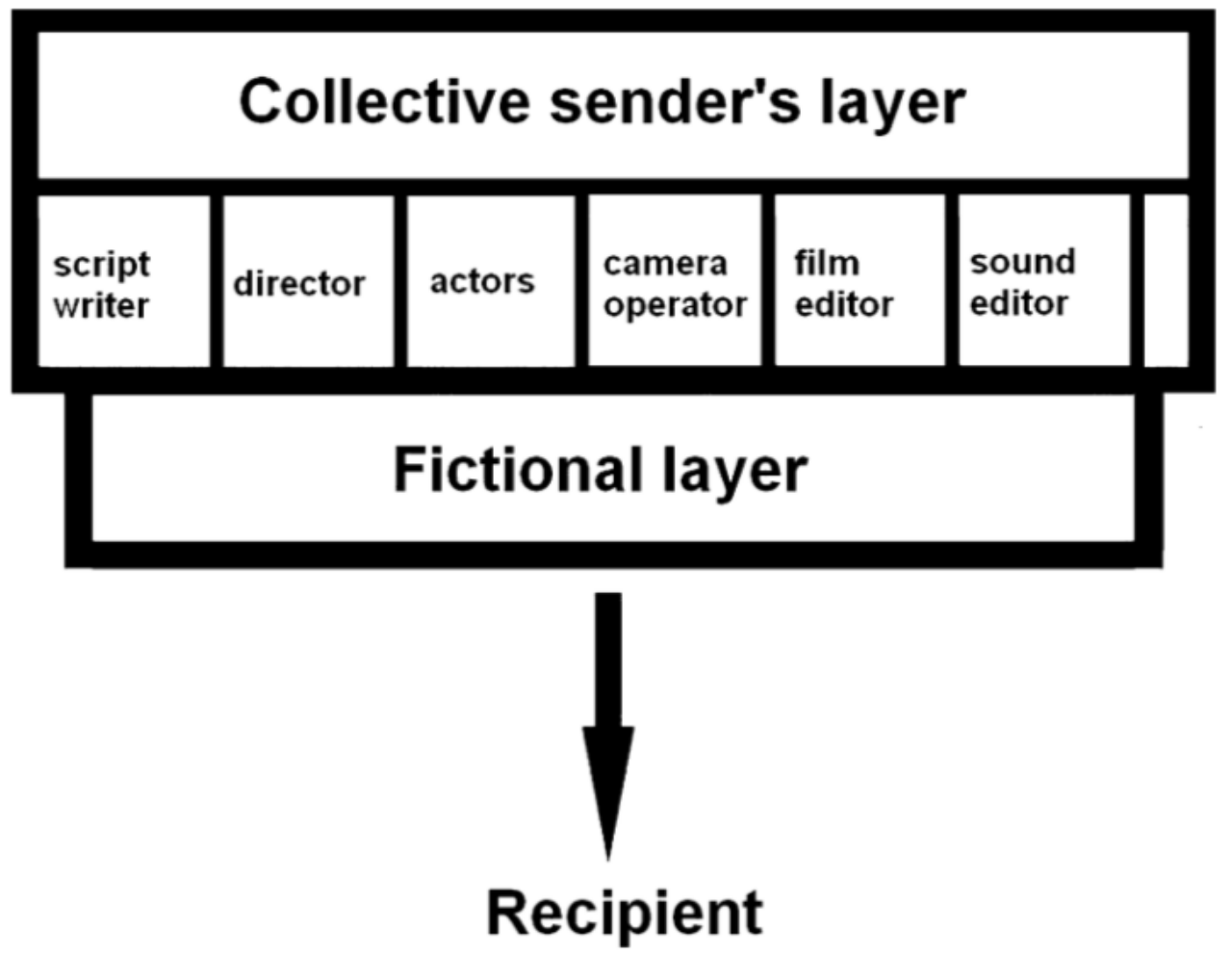

Dynel prend en compte la complexité auctoriale en jeu dans la création d'une série. Celle-ci est représentée par une auctorialité collective qui constitue un premier niveau de construction du sens de la série (collective sender's layer). À ce premier niveau s'ajoute un second niveau de sens construit par la fiction. Cette double articulation de la construction du sens est orientée vers le spectateur, qui en est le récepteur. Ce modèle a deux qualités : d'une part, il permet de refléter l'auctorialité collective et multiple d'une fiction sur écran; de l'autre, il permet de ne pas réduire le sens produit par la fiction à l'intention de ses auteurs, en proposant une articulation entre (au moins) deux niveaux (collective sender's layer et fictional layer) dans la construction du sens. Ce modèle unidirectionnel demeure cependant insatisfaisant dans la mesure où il implique une totale passivité du spectateur, conçu comme simple réceptacle d'un sens figé et préconstruit. Un modèle plus complet prendrait en compte le rôle actif du spectateur dans la construction du sens, ou pour le dire autrement, la dimension intersubjective de la relation entre le spectateur et la fiction à l'écran. Pour l'anthropolinguiste Duranti, le spectateur joue un rôle si central dans la construction du sens qu'il serait plus juste de l'envisager comme co-auteur de l'œuvre :

Interpretation (of texts, sounds etc.) is not a passive activity whereby the audience is just trying to figure out what the author meant to communicate. Rather, it is a way of making sense of what someone said (or wrote or drew) by linking it to a world or context that the audience can make sense of ${ }^{43}$.

C'est Bubel ${ }^{4445}$ qui propose le modèle le plus abouti de l'interaction entre spectateur et fiction à l'écran, à l'appui de travaux de linguiste interactionnelle en anthropologie linguistique, ethnométhodologie et psycholinguistique. Sa théorisation s'appuie notamment sur les travaux de Duranti sur le spectateur comme co-auteur, la notion de cadre de participation (participation framework) et la typologie des rôles de ceux qui écoutent (listener roles) tels qu'ils ont été proposés par le sociologue Goffman ${ }^{46}$ et le 
psychologue Clark $^{47}$, et enfin sur la notion de recipient design (ajustement de la production discursive à la représentation que le locuteur se fait des croyances, opinions et connaissances de l'interlocuteur) ${ }^{48}$; la notion de recipient design est, en ce sens, proche de celle de co-énonciation en linguistique énonciative).

Bubel (Figure 4) propose un modèle résolument interactionniste de la relation entre le spectateur et la fiction à l'écran, qu'elle nomme «screen-to-face interaction ». Ce modèle intègre l'auctorialité collective de l'œuvre de fiction, ainsi que la contribution du spectateur à la construction du sens, sous la forme de conjectures. Il prend également en compte le rôle spécifique du destinataire de la fiction à l'écran, qui s'adresse au spectateur en sa qualité de « ratified overhearer ».

Figure 4 : le modèle de l'interaction screen-to-face de Bubel ${ }^{49}$.

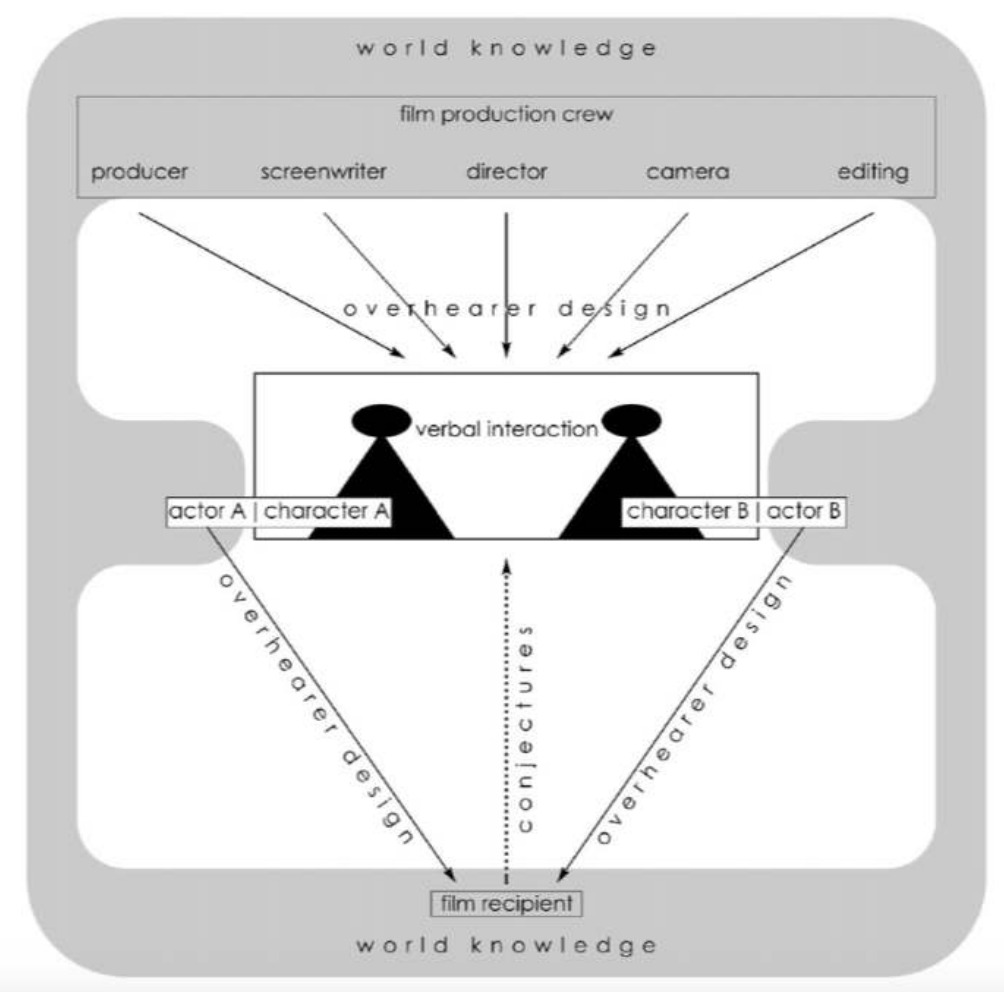

La conception du spectateur comme overhearer ratifié est particulièrement juste, néanmoins elle nécessite quelques explications car elle implique, en apparence, une formulation paradoxale, fruit de l'adaptation à l'interaction entre le spectateur et l'écran de catégories originellement conçues pour décrire la conversation ordinaire. Comme l'a montré le sociologue Goffman $(1976,1979)$, un individu qui entend un énoncé parlé peut occuper une grande diversité de rôles (listener roles) : si la parole lui est adressée, il fait partie des participants ratifiés de l'interaction ; si la parole ne lui est pas adressée, il est un participant non ratifié (c'est le cas, par exemple, de la situation très commune où l'on entend une conversation entre deux personnes dans les transports en commun). Clark développe une typologie des listener roles, représentée en Figure 5. 
Figure 5 : La typologie des listener roles de Clark ${ }^{50}$.

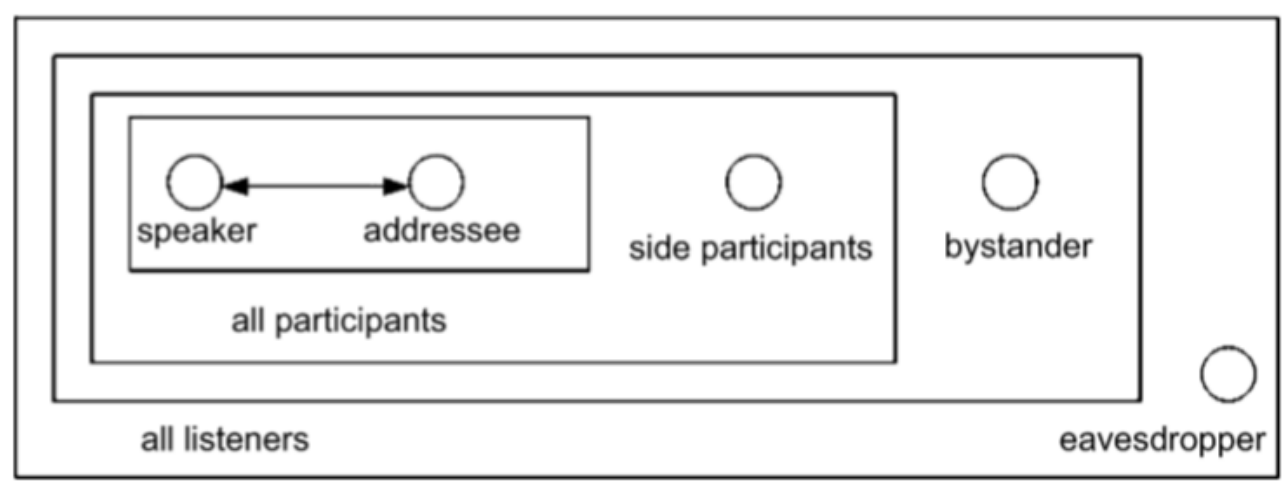

Lors d'une conversation à plusieurs, le locuteur (speaker) peut s'adresser un temps à un interlocuteur en particulier (addressee), par exemple pour lui poser une question. Même s'ils ne sont pas immédiatement concernés par la parole le temps de cet échange, les autres participants de la conversation (side participants) sont toujours considérés comme participants à part entière, ratifiés au même titre que le locuteur et l'interlocuteur. Parmi les participants non ratifiés, c'est-à-dire les overhearers, on distingue les témoins (bystanders), dont la présence dans l'environnement immédiat ne fait aucun doute pour les participants ratifiés, mais qui ne font pas partie de la conversation (c'est par exemple la place occupée par la personne qui entend une conversation dans les transports en commun). Les oreilles indiscrètes (eavesdroppers) sont les participants non ratifiés qui entendent une conversation sans que les participants ratifiés s'en rendent compte (par exemple si les participants ratifiés leurs tournent le dos, ou si l'oreille indiscrète espionne une conversation derrière une porte). En pratique, il existe un continuum entre certains de ces rôles, notamment entre bystander et eavesdropper, lorsque ceux qui croient ne pas être vus, ou entendus, le sont en réalité.

Lors de l'interaction entre le spectateur et l'écran, le spectateur est, en somme, le destinataire indirect des interactions représentées dans la fiction. Lorsque les personnages interagissent, le spectateur est placé en position d'overhearer des échanges dont il est témoin, sauf dans les cas d'apartés du personnage face caméra ${ }^{51}$, où il devient un temps destinataire direct de la parole, comme le note Bubel ${ }^{52}$. Dans tous les cas, c'est bien au spectateur que s'adresse la fiction à l'écran dans son ensemble : chaque action, chaque énoncé est destiné et anticipe les connaissances et les réactions du spectateur. Cette forme de recipient design se traduit notamment, on l'a vu, par le fait que le genre discursif du dialogue de fiction télévisée est à la fois moins vague et moins narratif que celui de la conversation spontanée, comme l'ont montré les travaux de Quaglio et Bednarek. La transposition des catégories de participation à l'interaction (participation framework) proposées par Goffman et Clark à la double articulation du discours à l'écran ou double-layering permet donc de définir le spectateur comme un overhearer ratifié.

\section{Le spectateur comme overhearer ratifié : une ressource créative}

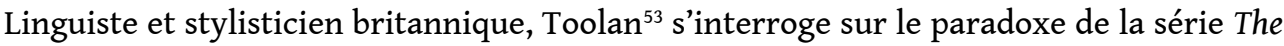
Wire (HBO, 2002-2008), comme le reflète le titre malicieux de son chapitre : " "I don't know what they're saying half the time, but I'm hooked on the series": Incomprehensible Dialogue and Integrated Multimodal Characterisation in The Wire ». 
Comment se fait-il, se demande Toolan, qu'une série où ce qui est dit est aussi inintelligible, même pour un locuteur natif, rencontre un tel engouement auprès des spectateurs? Notons que Toolan occulte complètement ici la question de la variation sociolinguistique, et en l'occurrence de l'intersection entre variation diatopique (d'une aire géographique à l'autre) diastratique (d'une classe sociale à l'autre) et diaphasique (d'une situation à l'autre pour un même locuteur), qui peuvent déjà, à elles seules, expliquer une grande part d'incompréhension mutuelle entre locuteurs natifs d'une même langue. Si ce point représente un manque indéniable dans son étude, Toolan propose, dans un premier temps, d'objectiver l'incompréhension du spectateur face à la série, en la mesurant. Il fait visionner deux fois à 24 participants ${ }^{54}$ un extrait vidéo de The Wire et un extrait d'une série britannique moins connue aux thématiques similaires, State of Play. Pendant le visionnage, il est fourni aux participants une transcription de chaque extrait (réalisée par Toolan lui-même et validée par deux relecteurs indépendants). Chaque transcription comporte 5 segments manquants de 10 mots en moyenne: les participants ont pour mission de remplir les segments manquants par une transcription la plus exacte possible. Par un système de notation sur une échelle de 1 à 5 , Toolan évalue la précision des transcriptions produites, par comparaison à la transcription cible qu'il en a faite. Les résultats sont les suivants : les transcriptions obtenues sont justes à $91 \%$ pour State of Play et à $82 \%$ pour The Wire, avec des scores en moyenne plus bas pour les participants non natifs. Pour Toolan, un score supérieur à $90 \%$ reflète une très bonne compréhension des contenus discursifs, tandis qu'un score inférieur à $85 \%$ reflète une compréhension parcellaire de ce qui est dit. En somme, The Wire est difficile à comprendre même pour des locuteurs natifs de l'anglais, en l'occurrence britanniques. Même si Toolan éclipse la question de l'incompréhension due à la variation sociolinguistique, la portée de sa démonstration n'en est pas pour autant amoindrie : l'incompréhension objective mise au jour par son protocole chez des locuteurs natifs d'anglais britannique n'empêche pas le succès de la série au RoyaumeUni et, plus largement, auprès de spectateurs dont l'anglais n'est pas la langue maternelle.

Comme le remarque Kozloff, l'opacité linguistique fait depuis longtemps partie des codes du film de gangsters; le sentiment d'étrangeté ou de frustration du spectateur face à des échanges qui ne sont que partiellement compréhensibles font partie intégrante de leur esthétique ${ }^{55}$. Toolan montre comment la difficulté de compréhension est en partie compensée par la répétition dans The Wire. Pour le montrer, Toolan procède à une analyse statistique par mots-clés (keyword analysis) de la transcription d'un épisode de The Wire proposées par des fans sur internet (fan transcript d'environ 5000 mots), qu'il compare à celle d'un épisode de House M.D. (Dr House; transcription de longueur équivalente), une série américaine en milieu hospitalier. Il ressort de cette analyse que les 13 mots les plus fréquents représentent 4,33\% pour House M.D. mais 6,38\% pour The Wire. Ce résultat témoigne d'une moindre diversité lexicale dans The Wire et donc d'un plus fort taux de répétition. Pour Toolan, la répétition est un procédé inscrit dans le script même de la série pour compenser l'opacité linguistique et favoriser la compréhension $\mathrm{du}$ spectateur («scripted repetition as aid to comprehension " ${ }^{56}$ ). L'opacité linguistique est, bien sûr, également compensée par les indices vocaux et visuels auxquels les discours s'intègrent en contexte ${ }^{57}$. fait d'inscrire la série dans la lignée des films de gangsters. Comme le souligne Toolan, 
la force de The Wire est de positionner, par le biais de l'incompréhensibilité, le spectateur aux côtés de personnages qui ne cessent de s'épier les uns les autres. Le policier qui met sur écoute les gangs se retrouve dans la même position que le spectateur : un overhearer qui tente de déchiffrer le sens des mots de ceux qu'il écoute. Ce dispositif où la fiction met en abyme l'expérience, bien réelle, du spectateur, crée une continuité entre espace réel et espace fictionnel, qui favorise une identification forte du spectateur aux personnages, devenus ses doubles fictionnels.

\section{Conclusion}

des relations en jeu entre le spectateur et la série : l'interaction screen-to-face a lieu dans un espace bien réel où le spectateur occupe la place spécifique d'overhearer ratifié, qui participe de manière active à la construction intersubjective du sens, comme l'a proposé Bubel à l'appui des travaux de Goffman, Clark et Duranti. spectateur. L'opacité linguistique vise à créer l'incompréhension du spectateur, de manière à le positionner aux côtés des personnages qui s'épient et mettent leurs ennemis sur écoute. La fiction fait alors écho à l'expérience réelle du spectateur, créant une continuité entre espaces réel et fictionnel qui favorise l'identification du spectateur aux personnages.

\section{BIBLIOGRAPHIE}

BEDNAREK, Monika, The Language of Fictional Television: Drama and Identity, Londres et New York, Continuum, 2010.

BEDNAREK, Monika, « The language of fictional television: a case study of the 'dramedy' Gilmore Girls ", English Text Construction, Vol. 4, No. 1, 2011a, p. 54-83.

BEDNAREK, Monika, " The stability of the televisual character: A corpus stylistic case study ", in Roberta Piazza, Monika Bednarek et Fabio Rossi, éd., Telecinematic Discourse: Approaches to 
Language in Fictional Cinema and Television, Amsterdam et Philadelphie, John Benjamins, 2011b, p. 185-204.

BELL, Allan, The Language of News Media, Oxford, Blackwell, 1991.

BUBEL, Claudia, The Linguistic Construction of Character Relations in TV Drama: Doing Friendship in Sex and the City, Sarrebruck, Universitat des Saarlandes, 2006.

BUBEL, Claudia, « Film audiences as overhearers », Journal of Pragmatics 40, 2008, p. 55-71.

BURGER, Harald, Sprache der Massenmedien, Berlin, de Gruyter, 1984.

BURGER, Harald, Das Gesprach in den Massenmedien, Berlin, de Gruyter, 1991.

CLARK, Herbert, SCHAEFER, Edward, « Dealing with overhearers », in Herbert Clark, éd., Arenas of Language Use, Chicago, University of Chicago Press, 1992, p. 248-273.

CLARK, Herbert, Using Language, Cambridge, Cambridge University Press, 1996.

DESCOLA, Philippe, Par-delà nature et culture, Paris, Gallimard, 2005.

DÍAZ CINTAS, Jorge, «Introduction - Audiovisual translation: an overview of its potential. », in Jorge Díaz Cintas, éd., New Trends in Audiovisual Translation, Bristol, Multilingual Matters, 2009, [p. 1-20], p. 7-8.

DURANTI, Alessandro, « The audience as co-author: an introduction », Text 6, 1986, p. 239-247.

DYNEL, Marta, «You talking to me?" The viewer as a ratified listener to film discourse ", Journal of Pragmatics 43, 2011, p. 1628-1644.

FIELD, Syd, The Screenwriter's Workbook, New York, Dell, 1984, p. 70.

GOFFMAN, Erving, « Replies and responses », Language in Society 5, 1976, p. 257-313.

GOODWIN, Charles, DURANTI, Alessandro, « Rethinking Context: An Introduction », in Alessandro Duranti et Charles Goodwin, éd., Rethinking Context, Cambridge et New York, Cambridge University Press, 1992, p. 1-42.

KOZLOFF, Sarah, Overhearing Film Dialogue, Berkeley, University of California Press, 2000.

LORENZO-DUS, Nuria, " “You're barking mad, I'm out”: Impoliteness and broadcast talk », Journal of Politeness Research 5, 2009, p. 159-187.

MCINTYRE, Dan, « Integrating Multimodal Analysis and the Stylistics of Drama: a Multimodal Perspective on Ian McKellen's Richard III ", Language and Literature, Vol. 17, No. 4, 2008, p. 309-34.

O'KEEFFE, Anne, Investigating Media Discourse, Londres, Routledge, 2006.

PEARSON, Roberta, « Anatomising Gilbert Grissom. The structure and function of the televisual character. ", in Reading CSI. Crime TV under the Microscope, éd. Michael Allen, Londres et New York, I.B. Tauris, 2007, p. 39-56.

PIAZZA, Roberta, BEDNAREK, Monika, ROSSI, Fabio, "Introduction: analyzing telecinematic discourse", in Roberta Piazza, Monika Bednarek et Fabio Rossi, éd., Telecinematic Discourse: Approaches to Language in Fictional Cinema and Television, Amsterdam et Philadelphie : John Benjamins, 2011, p. 1-17.

QUAGLIO, Paulo, Television Dialogue. The Sitcom Friends vs. Natural Conversation, Amsterdam et Philadelphie, John Benjamins, 2009. 
QUAGLIO, Paulo, «Television dialogue and natural conversation: Linguistic similarities and functional differences ", in Annelie Ädel et Randi Reppen, éd., Corpora and Discourse. The Challenges of Different Settings, Amsterdam et Philadelphie, John Benjamins, 2008, p. 189-210.

RICHARDSON, Kay, « Multimodality and the study of popular drama », Language and Literature Vol. 19, No. 4, 2010, p. 378-395.

SACKS, Harvey, SCHEGLOFF, Emanuel JEFFERSON, Gail, « A Simplest Systematics for the Organization of Turn-Taking for Conversation », Language, Vol. 50, No. 4, Part 1, 1974, p. 696-735.

SCANNELL, Paddy, « Introduction: the relevance of talk », in Paddy Scannell, éd., Broadcast Talk, Londres, Sage, 1991, p. 1-13.

SHANNON, Claude, WEAVER, Warren, A Mathematical Model of Communication, Urbana, University of Illinois Press, 1949.

SHORT, Mick, « Discourse analysis and the analysis of drama. », in Ronald Carter et Paul Simon, éd., Language, Discourse and Literature. An Introductory Reader in Discourse Stylistics, Londres et New York, Routledge, 1989, p. 139-168.

SHORT, Mick, « Discourse analysis and drama » in The Encyclopedia of Language and Linguistics, éd. Ron Asher, Oxford, Pergamon, 1994, p. 949-952.

TOOLAN, Michael, « “I don't know what they're saying half the time, but I'm hooked on the series" Incomprehensible dialogue and integrated multimodal characterisation in The Wire ", in Roberta Piazza, Monika Bednarek \& Fabio Rossi, éd., Telecinematic Discourse: Approaches to Language in Fictional Cinema and Television, Amsterdam et Philadelphie, John Benjamins, 2011, p. 161-183.

VORHAUS, John, The Comic Toolbox: How to be Funny Even if You're Not, Los Angeles, Silman-James Press, 1994.

\section{NOTES}

1. Charles Goodwin, Alessandro Duranti, «Rethinking Context: An Introduction », in Rethinking Context, éd. Alessandro Duranti et Charles Goodwin, Cambridge et New York, Cambridge University Press, 1992, p. 1-42.

2. Harald Burger, Das Gesprach in den Massenmedien, Berlin, de Gruyter, 1991; Allan Bell, The Language of News Media, Oxford, Blackwell, 1991; Paddy Scannell, « Introduction: the relevance of talk », in Broadcast Talk, éd. Paddy Scannell, Londres, Sage, 1991, p. 1-13; Anne O'Keeffe, Investigating Media Discourse, Londres, Routledge, 2006.

3. Harald Burger, Sprache der Massenmedien, Berlin, de Gruyter, 1984, p. 44 et suivantes.

4. Nuria Lorenzo-Dus, « "You're barking mad, I'm out”: Impoliteness and broadcast talk », Journal of Politeness Research Vol. 5, No. 2, 2009, p. 159-187.

5. Herbert Clark et Edward Schaefer, «Dealing with overhearers ", in Arenas of Language Use, éd. Herbert Clark, Chicago, University of Chicago Press, 1992, p. 248-273.

6. Roberta Piazza, Monika Bednarek, Fabio Rossi, "Introduction: analyzing telecinematic discourse", in Telecinematic Discourse: Approaches to Language in Fictional Cinema and Television, éd. Roberta Piazza, Monika Bednarek, Fabio Rossi, Amsterdam et Philadelphie, John Benjamins, p. $1-17$.

7. « Since a large percentage of films and television programmes consumed by viewers worldwide are originally produced in the United States it seems legitimate to expect that they will exert a certain degree of influence both in the language - usually via translation - and in the attitudes of 
millions of people across the globe. Whilst aiming to mirror society, audiovisual productions invite their audiences to find resemblances in the characters they see on screen in a process of identification, which in turn triggers a mimetic attitude in some viewers. ». Jorge Díaz Cintas, "Introduction - Audiovisual translation: an overview of its potential», in New Trends in Audiovisual Translation, éd. Jorge Díaz Cintas, Bristol, Multilingual Matters, 2009, p. 7-8, [p. 1-20].

8. Monika Bednarek, «The language of fictional television: a case study of the 'dramedy' Gilmore Girls ", English Text Construction, Vol. 4, No. 1, 2011a, p. 54-83.

9. «From the perspective of English/American Studies, research on television dialogue is important on the one hand because television programmes are cultural products just like novels and films, and on the other hand because the scripted dialogue of fictional television series can have a significant impact on learners of English in non-English-speaking countries (...) and television programmes may be used in the English language classroom (...). » Bednarek, 2011a, p. 3.

10. Kay Richardson, "Multimodality and the study of popular drama ", Language and Literature Vol. 19, No. 4, 2010, p. 378-395.

11. Dan McIntyre, «Integrating Multimodal Analysis and the Stylistics of Drama: a Multimodal Perspective on Ian McKellen's Richard III ", Language and Literature, Vol. 17, No. 4, 2008, p. 309-34. 12. Sarah Kozloff, Overhearing Film Dialogue, Berkeley, University of California Press, 2000.

13. «Films and TV dramas are indeed multimodal texts. Narrative cinema is a mode in its own right, but a complex one which exploits other modes, such as dialogue, that are not unique to cinema. The complexity of the articulations in particular textual instances will always prohibit formal analysis of any given movie in its totality, whatever that might mean, and there is no specific apparatus currently fit for purpose. Multimodal semiotics is too general; traditional stylistics too linguistic and traditional film studies too narratological/visual. [...] In this context there is no need to apologize for adopting a logocentric approach, just so long as [...] there remains space for approaches oriented to other aspects of production and scope for collaboration between practitioners of different approaches ». Richardson, p. 390.

14. Piazza, Bednarek, Rossi, p. 9.

15. Claudia Bubel présente Syd Field comme l'un des plus influents professeurs d'écriture de scénario aux Etats-Unis (voir Claudia Bubel, The Linguistic Construction of Character Relations in TV Drama: Doing Friendship in Sex and the City, Sarrebruck, Universitat des Saarlandes, 2006).

16. Syd Field, The Screenwriter's Workbook, New York, Dell, 1984, p. 70.

17. Paulo Quaglio, Television Dialogue. The Sitcom Friends vs. Natural Conversation, Amsterdam et Philadelphie, John Benjamins, 2009.

18. Quaglio, 2009, p. 13.

19. Les travaux de Douglas Biber font autorité dans le champ de la linguistique de corpus. Biber est le principal éditeur d'une grammaire de référence fondée sur l'étude de grand corpus, la Longman Grammar of Spoken and Written English, éditée pour la première fois en 1999.

20. Douglas Biber, « avant-propos », in Paulo Quaglio, p. xiii.

21. Si Quaglio et Biber utilisent, sans le problématiser, le terme de conversation " naturelle », je lui préfère celui de conversation "spontanée». En effet, tout corpus d'interactions réelles constitue une construction, où les participants sont conscients d'être enregistrés, même si cela bien souvent n'affecte que marginalement la forme de leurs productions. Plus largement, la notion de «naturel » est, elle aussi, une catégorie construite. Voir Philippe Descola, Par-delà nature et culture, Paris, Gallimard, 2005.

22. Quaglio, 2009, op. cit.

23. Monika Bednarek, The Language of Fictional Television: Drama and Identity, Londres et New York, Continuum, 2010.

24. «[Fan transcripts are] fairly accurate and very detailed, including several features that scripts are not likely to present: hesitators, pauses, repeats, and contractions ». Paulo Quaglio, 
«Television dialogue and natural conversation: Linguistic similarities and functional differences ", in Corpora and Discourse. The Challenges of Different Settings, éd. Annelie Ädel et Randi Reppen, Amsterdam et Philadelphie, John Benjamins, 2008, p. 191-192, [p. 189-210].

25. "The American conversation subcorpus of the Longman Grammar Corpus has approximately $4,000,000$ words. Carefully designed to be representative of American English conversation, it includes a wide range of settings (e.g. park, family home, classroom), types of interactions (e.g., casual conversations, task-related, telephone conversations), geographical regions (i.e., states, cities) and speaker attributes (e.g. age, sex, occupation, years of schooling). The informants who took part in the collection of this corpus tape-recorded all of their conversations over a period of a week. These recordings were then transcribed and saved electronically for linguistic research purposes. Each of the conversations (referred to as texts) includes headers, preceding the transcriptions, containing the information mentioned above (e.g. settings, types of interactions) ». Quaglio, 2009, p. 36.

26. Quaglio, 2009, p. 139.

27. Bednarek, 2011a, op. cit.

28. «n-grams are register/genre/text- type sensitive (...), that is, that they can discriminate between text-types » Bednarek, 2010, op. cit.

29. Bednarek, 2011a, p. 16.

30. Nous n'y procéderons pas ici par manque de place, mais cette idée mériterait à elle seule un développement détaillé sur la question du statut fictionnel des séries, et en particulier sur la tension entre fiction et réalité/réalisme des interactions à l'écran.

31. Monika Bednarek, "The stability of the televisual character: A corpus stylistic case study », in Telecinematic Discourse: Approaches to Language in Fictional Cinema and Television, éd. Roberta Piazza, Monika Bednarek et Fabio Rossi, Amsterdam et Philadelphie, John Benjamins, 2011b, p. 185-204.

32. Roberta Pearson, « Anatomising Gilbert Grissom. The structure and function of the televisual character ", in Reading CSI. Crime TV under the Microscope, éd. Michael Allen, Londres et New York, I.B. Tauris, 2007, p. 56. [p. 39-56].

33. John Vorhaus, The Comic Toolbox: How to be Funny Even if You're Not, Los Angeles, Silman-James Press, 1994, p. 56.

34. Citation tirée d'une interview d'Amy Sherman-Palladino par Scott Tobias pour The Onion A.V. Club datée du 9 février 2005, citée par Bednarek, 2011b, p. 9.

35. Bednarek, 2011b, op. cit.

36. Bednarek, 2011b, p. 193.

37. Mick Short, "Discourse analysis and the analysis of drama", in Language, Discourse and Literature. An Introductory Reader in Discourse Stylistics, éd. Ronald Carter et Paul Simon, Londres et New York, Routledge, 1989, p. 139-168.

38. Claude Shannon, Warren Weaver, A Mathematical Model of Communication, Urbana, University of Illinois Press, 1949.

39. Mick Short, «Discourse analysis and drama », in The Encyclopedia of Language and Linguistics, éd. Ron Asher, Oxford, Pergamon, 1994, p. 949-952.

40. Short, 1989, p. 149.

41. Marta Dynel, " "You talking to me?" The viewer as a ratified listener to film discourse", Journal of Pragmatics, Vol. 43, No. 6, 2011, p. 1628-1644.

42. Dynel, 2011, p. 1635.

43. Alessandro Duranti, «The audience as co-author: an introduction », Text, Vol. 6, No. 3, 1986, p. 243-244 [p. 239-247].

44. Bubel, 2006, op. cit.

45. Claudia Bubel, «Film audiences as overhearers », Journal of Pragmatics, Vol. 40, No. 1, 2008, p. $55-71$.

46. Erving Goffman, « Replies and responses », Language in Society, Vol. 5, No. 1, 1976, p. 257-313. 
47. Herbert Clark, Using Language, Cambridge, Cambridge University Press, 1996.

48. "By 'recipient design' we refer to a multitude of respects in which talk by a party in conversation is constructed or desgned in ways which display an orientation and sensitivity to the particular other(s) who are the co-participants ». Harvey Sacks, Emanuel Schegloff, Gail Jefferson, "A Simplest Systematics for the Organization of Turn-Taking for Conversation ", Language, Vol. 50, No. 4, Part 1, 1974, p. 727 [p. 696-735].

49. Bubel, 2008, art. cit.

50. Clark, 1996, p. 14.

51. Il s'agit d'un dispositif fréquemment utilisé par le personnage principal dans certaines séries comme Sex and the City, comme le note Bubel, ou, plus récemment dans Fleabag.

52. Bubel, 2008, art. cit.

53. Michael Toolan, « “I don't know what they're saying half the time, but I'm hooked on the series" Incomprehensible dialogue and integrated multimodal characterisation in The Wire ", in Telecinematic Discourse: Approaches to Language in Fictional Cinema and Television, éd. Roberta Piazza, Monika Bednarek, Fabio Rossi, Amsterdam et Philadelphie, John Benjamins, 2011, p. 161-183.

54. 20 participants natifs et 4 non-natifs. Toolan parle de "young educated adults » (p. 172); il s'agit peut-être de certains de ses étudiants; le lecteur suppose que les natifs sont des britanniques.

55. Kozloff, p. 215.

56. Toolan, p. 173

57. Toolan, p. 177-181.

\section{RÉSUMÉS}

Les séries plaisent-elles parce que les personnages parlent comme le spectateur, reflétant ainsi son univers? Cet article propose un tour d'horizon des approches linguistiques des séries, autour de la notion centrale d'espace. Deux approches se sont particulièrement saisies des séries, la linguistique de corpus et la linguistique interactionnelle, autour de trois enjeux centraux: la représentation du réel, la définition des personnages et l'interaction avec le spectateur. À partir d'analyses semi-automatisées de transcriptions des dialogues des séries, la linguistique de corpus en caractérise le genre discursif. La linguistique interactionnelle théorise l'enchâssement des deux espaces d'interaction mis en jeu : l'interaction réelle entre le spectateur et la série, qui se construit par le biais de l'interaction fictionnelle entre les personnages.

Comme le montrent Quaglio et Bednarek, le genre discursif de la série est plus informel, plus émotionnel, moins vague, et moins narratif que la conversation spontanée. La stabilité langagière des personnages au fil des saisons est centrale pour construire la stabilité de l'univers fictionnel. Les pratiques langagières et sémiotiques des personnages mettent en jeu les attentes et les cadres interprétatifs socioculturels du spectateur pour construire des types.

L'espace interactionnel spectateur-fiction a donné lieu à de multiples théorisations; le modèle d'interaction screen-to-face proposé par Bubel est le plus abouti. Celui-ci intègre les notions de participation framework proposée par Goffman et Clark, celle de recipient design proposée par Sacks et ses collègues, ainsi que les travaux anthropologiques de Duranti sur le rôle actif du spectateur. Toolan montre que l'opacité linguistique dans une série comme The Wire est sa force, car la série 
joue sur le dispositif d'interaction screen-to-face, en positionnant un spectateur en proie à de constantes difficultés de compréhension aux côtés de personnages qui ne cessent de s'épier.

Do characters in TV shows use language and interact the way people do? And is that why we like watching TV shows? This article proposes an overview of linguistic approaches to TV series, based on the central notion of space. Linguistic analyses of TV series have mostly been proposed by corpus linguistics and interactional linguistics, with a focus on three main issues: the relationship between real life and fictional discourse, the definition of characters, and the relationship between represented and interactive participants. Corpus linguistics relies on semiautomated analyses of scripts to characterize the linguistic genre of TV series dialogue. Interactional linguistics proposes theories of the interaction between the viewer and the series, which is constructed via the fictional interaction between characters.

As shown by Quaglio and Bednarek, the discursive genre of TV series is more informal, more emotional, less vague, and less narrative than spontaneous conversation. The stability of a character's language use across seasons is crucial in maintaining the stability of the fictional world. Characters' use of language and signs mobilize the spectator's socio-cultural expectations and interpretation frameworks so as to construct types.

Several theories of the real interaction space involving the viewer and the series have been proposed. The most comprehensive one is Bubel's model of screen-to-face interaction. It includes the notions of participation framework proposed by Goffman and Clark, Sacks and colleagues' recipient design and Duranti's anthropological work on the spectator's active role. Toolan shows how the success of The Wire is paradoxically explained by its linguistic opacity, which plays with screen-to-face interaction by positioning the viewer alongside the characters who are having a hard time understanding others while spying on them.

\section{INDEX}

Mots-clés : linguistique interactionnelle, linguistique de corpus, Friends, Gilmore Girls, Sur écoute

Keywords : interactional linguistics, corpus linguistics, Friends, Gilmore Girls, Wire (The)

\section{AUTEUR}

\section{CAMILLE DEBRAS}

Camille Debras est maître de conférences en linguistique anglaise à l'Université Paris Nanterre. Dans ses recherches en linguistique interactionnelle sur la multimodalité de la langue, elle étudie la manière dont les locuteurs intègrent les gestes au discours dans la construction séquentielle et intersubjective du sens, dans des corpus variés (conversation ordinaire, interaction multipartite, interactions adulte-enfant, interactions natif-non natif). Ses intérêts de recherche incluent l'anthropologie linguistique, l'analyse du discours, la communication non verbale et la sémiotique multimodale.

Camille Debras is an associate professor in English linguistics at Université Paris Nanterre. Her research in the field of interactional linguistics and multimodality focuses on how speakers use gestures in speech sequences to create intersubjective meaning in varied corpora based on authentic interactions. Her other interests include linguistic anthropology, discourse analysis, non-verbal communication and multimodal semiotics. 